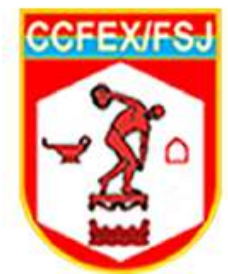

Revista de Educação Física

Joumal of Physical Education

Home page: unn revistadeeducacsolisica.00m

Artigo Original

Original Article

\title{
0 uso do ciclo alongamento-encurtamento em saltos de atletas de handebol e praticantes de musculação do sexo feminino
}

\section{Use Stretch-Shortening Cycle in Leaps Handball Athletes and Female Bodybuilders}

Hyago Bernardes da Rosa ${ }^{1}$; Vinicius da Silva Lessa de Oliveira ${ }^{1}$; Lucas Souza Santos ${ }^{1}$; Samuel Klippel Prusch ${ }^{1}$; Igor Martins Barbosa ${ }^{1}$; Luiz Fernando Cuozzo Lemos ${ }^{1 \S} \mathrm{PhD}$

Recebido em: 14 de março de 2016. Aceito em: 13 de junho de 2016

Publicado online em: 29 de setembro de 2016.

\section{Resumo}

Introdução: O handebol é caracterizado como um esporte de cunho coletivo, possuindo grande variedade de movimentações e seus ganhos propiciam melhoramentos nas qualidades físicas e funcionais. Por outro lado, a musculação, que é amplamente praticada atualmente, possibilita muitos ganhos, porém, proporciona baixo melhoramento funcional. 0 Ciclo AlongamentoEncurtamento (CAE) está baseado no fato de o músculo realizar ativamente uma ação, sendo parte dessa energia armazenada nos componentes elásticos e reutilizada na próxima ação muscular.

Objetivo: Comparar a utilização do CAE na potência dos membros inferiores entre os grupos analisados.

Métodos: A amostra foi constituída por 16 atletas de handebol (GH) e 12 praticantes de musculação (GM), ambos do sexo feminino. Foi mensurada a potência através da plataforma de contato e atividade eletromiográfica dos músculos: bíceps femoral, reto femoral, gastrocnêmio medial e vasto lateral, através de testes de saltos verticais: Squat Jump e Counter Movement Jump.

Pontos-Chave Destaque

- O tipo de treinamento está

relacionada ao armazenamento

de força elástica

- Houve diferença na economia

de ativação elétrica do músculo

vasto lateral entre os grupos

- Atletas de handebol

aproveitam a força elástica

para maior economia em saltos

verticais

Resultados: As diferenças estatísticas significativas encontradas entre os grupos foram: o tempo de prática para o GH foi maior, o tempo de prática semanal para o GM foi superior e o percentual de utilização do CAE que para o GH foi de -2,63 e no GM foi de 13,32, para o músculo vasto lateral.

Conclusão: Por meio dos resultados obtidos por testes de saltos verticais, o GH utilizou com maior eficiência a força elástica, apresentando superioridade na economia de ativação do vasto lateral. Portanto, as praticantes de musculação são menos econômicas, possivelmente por seu treinamento isolado.

Palavras-chave: handebol, treinamento de força, ciclo alongamento-encurtamento do ciclo, biomecânica, eletromiografia.

\begin{abstract}
Introduction: Handball is a sport of collective nature, featuring a great variety of moves and its gains provide improvements in physical and functional qualities. However, bodybuilding, which is commonly practiced nowadays, allows many gains, nonetheless, provides low functional improvement. The Stretch-Shortening Cycle (SSC) is based on the fact that the muscle acts actively, with a part of this energy stored in the elastic components and reutilized on the next muscular action.
\end{abstract}

\footnotetext{
${ }^{\S}$ Autor correspondente: Luiz Fernando Cuozzo Lemos - e-mail: luizcanoagem@yahoo.com.br. Afiliações: ${ }^{1}$ Faculdade Cenecista de Osório-RS.
} 
Objective: To compare the use of the SSC on the potency of the inferior limbs amongst the analyzed groups.

Methods: The sample was built by 16 handball athletes (GH) and 12 bodybuilding practitioners (GM), both of the womankinds. The potency was measured through the muscular contact and electromyographic activity platform: Biceps femoris, rectus femoris, medial gastrocnemius and vastus lateralis, with vertical jumps: Squat Jump e Counter Movement Jump.

Results: The significant statistical differences found between the group were: the practice time for the GH was higher, the weekly practice time for the GM was higher and the usage percentage of the SSC for the GH was of -2.63 and in the GM was of 13.32 , for the vastus lateralis muscle.

Conclusion: With the results obtained with tests of vertical jumps, the GH used the elastic force more efficiently, displaying superiority on the activation economy of the vastus lateralis. Therefore, the bodybuilding practitioners are less economic, possibly due to their isolated training.

\section{Keypoints}

- The type of training is related

to the tensile strength of

storage

- There was a difference in the

electrical activation of the

economy of the vastus lateralis

muscle among groups

- Handball athletes benefit from

the tensile strength for greater

economy in vertical jumps

Keywords: handball, strength training, stretch-shortening cycle, biomechanics, electromyography

\section{0 uso do ciclo alongamento-encurtamento em saltos de atletas de handebol e praticantes de musculação do sexo feminino}

\section{Introdução}

Assim como em outros esportes de cunho coletivo, o handebol é caracterizado por uma variedade de movimentações, manipulação de bola e de interação entre os outros praticantes da modalidade (1). O treinamento dessa modalidade proporciona ganhos em qualidades físicas específicas e funcionais (2).

Por outro lado, o treinamento de força amplamente praticado nos dias atuais, embora possibilite muitos ganhos e faça parte de períodos preparatórios de diversos esportes, como o handebol, por exemplo, é criticado por estudiosos pelo fato de, quando praticado isoladamente, proporciona baixos índices de melhoramentos funcionais. Em outras palavras, o beneficio (transmissão dos resultados) para as atividades da vida diária é menor que outras modalidades (3).

Uma informação que poderia identificar essa menor funcionalidade nos ganhos realizados pelo treinamento de força em exercícios localizados específicos (musculação) é um menor aproveitamento da energia potencial elástica, por exemplo, em testes de saltos.
O mecanismo que explica a utilização da energia elástica é chamado de Ciclo Alongamento-Encurtamento (CAE). O referido mecanismo está baseado no fato de o músculo realizar ativamente uma ação, sendo parte da energia armazenada nos componentes elásticos e reutilizada na próxima ação muscular, o qual propicia uma maior potência quando a ação muscular excêntrica é imediatamente seguida por uma ação muscular concêntrica (4).

Para quantificação do nível de utilização CAE em saltos é utilizada uma equação que em os valores de dois tipos de saltos são necessários. O primeiro valor é proveniente do salto no chamado Squat Jump (SJ). O segundo valor é oriundo do counter moviment jump (CMJ) (5).

$\mathrm{O}$ uso da eletromiografia (EMG) juntamente aos testes de saltos verticais vem servindo como uma das principais ferramentas para pesquisas atuais, e, com o uso desse instrumento é possível analisar as amplitudes de sinais da atividade elétrica que ocorrem nas membranas logo abaixo dos eletrodos superficiais a pele (6). 
Este estudo tem por objetivo comparar a utilização do Ciclo AlongamentoEncurtamento (CAE) na potência dos membros inferiores (MMII) verificados pelos testes de saltos verticais $\mathrm{SJ}$ e CMJ em dois grupos ativos (handebol e musculação).

\section{Métodos}

$\mathrm{O}$ termo de consentimento livre e esclarecido (TCLE) foi assinado por cada participante. $\mathrm{O}$ projeto foi aprovado pelo Comitê de Ética em Pesquisa da Faculdade Cenecista de Osório - FACOS sob o protocolo $\mathrm{n}^{\mathrm{o}}$ 50191115.7.00005591.

\section{Amostra e Critérios de Elegibilidade}

A amostra foi constituída por dois grupos, conforme a Tabela 1, destacando que essa divisão foi feita intencionalmente, para obter a comparação do CAE na potência dos MMII. Os critérios de inclusão foram: não ter sofrido lesão osteomioarticular nos últimos seis meses, estar praticando a modalidade específica de cada grupo por pelo menos seis meses. Como critérios de exclusão foi considerado que os participantes não poderiam apresentar qualquer tipo de distúrbio vestibular, alteração visual sem correção, diabetes, lesões no sistema musculoesquelético ou dor lombar.

\section{Coleta de dados}

Para a aquisição dos sinais eletromiográficos, tanto durante os saltos como para a obtenção das Contrações Voluntárias Isométricas Máximas (CVIM), foi utilizado a eletromiografia de superfície (EMG). Os músculos avaliados foram o vasto lateral (VL), reto femoral (RF) e bíceps femoral (BF) e gastrocnêmio medial (GM). Foi utilizado um eletromiógrafo Miotec (Porto Alegre, Brasil), com quatro canais de entrada operando na frequência de $2000 \mathrm{~Hz}$. O sinal captado pelo eletromiógrafo foi gravado em um computador no software Miograph (Miotec Equipamentos Biomédico Ltda, Brasil) para posterior análise.

Foram utilizados eletrodos de superfície na configuração bipolar (AgCL3; modelo Meditrace, da marca $3 \mathrm{M})$, posicionados paralelamente e separados por $20 \mathrm{~mm}$. Os eletrodos foram posicionados longitudinalmente e na direção das fibras musculares de acordo com as recomendações da SENIAM (Surface EMG for Non-Invasive Assessment of Muscles) (7). Para facilitar a visualização do local do posicionamento dos eletrodos, foi solicitado ao avaliado realizar uma contração dos referidos músculos com objetivo de identificação do ventre muscular. Um eletrodo de referência foi posicionado sobre a face anterior da tíbia. Antes da fixação dos eletrodos, realizou-se a tricotomia, abrasão e limpeza da pele com algodão e álcool para remover as células mortas e a oleosidade, a fim de reduzir a impedância.

Para normalização do sinal eletromiográfico se realizou (CIVM), nos músculos: bíceps femoral, reto femoral e vasto lateral, e gastrocnêmico medial, conforme Correa et. al. (8) e Cardoso et. al., (9).

Os sinais brutos de EMG foram filtrados por um filtro passa-banda de 20 a $500 \mathrm{~Hz}$ de $5^{\mathrm{a}}$ ordem para atenuarem variações nos dados.

Antes da realização das tentativas dos saltos, as participantes fizeram um aquecimento na esteira rolante durante cinco minutos (velocidade de $6,5 \mathrm{Km} / \mathrm{h}$ ). Após isso, foram realizadas cinco tentativas dos saltos Squat Jump (SJ) e do Counter Movement Jump (CMJ), para cada participante. Entre cada tentativa, foram dados 30 segundos de descanso. Após a aquisição dos saltos válidos, foi realizada a média dos dois melhores saltos de cada categoria.

Utilizou-se também uma plataforma de contato CEFISE $\AA$, conectada ao sistema para medida de salto Jump System ${ }^{\circledR}$, para obter o pico de altura máxima dos saltos SJ e CMJ, além da potência gerada pelos sujeitos.

A execução do CMJ se deu com o indivíduo de pé com meias ou descalço sobre uma plataforma de contato, com o peso distribuído uniformemente sobre ambos os membros inferiores. As mãos foram colocadas sobre os quadris, onde ficaram durante todo o teste. Assim, o avaliado antes de saltar começou em uma posição em pé e quando tudo estava pronto, agachou-se flexionando os joelhos em um ângulo de 90 graus, imediatamente antes de saltar verticalmente o mais alto possível, mantendo os joelhos em extensão durante todo o voo e caindo com os dois pés ao mesmo tempo. O salto foi ser executado com ambos os pés, sem passos iniciais (10). Já o 
SJ, segue os mesmos padrões do CMJ, porém exigiu que o indivíduo começasse o movimento partindo de uma posição agachada com os joelhos flexionados a aproximadamente 90 graus, imóvel, com o tronco ereto, olhando para frente e tendo as mãos sobre os quadris. O avaliado efetuou uma forte e rápida extensão dos membros inferiores sem contra movimento e manteve as mãos no quadril. $\mathrm{O}$ desempenho máximo obtido coincidiu com um salto vertical o mais alto possível (10).

$\mathrm{Na}$ realização de ambos os testes, os saltos seriam invalidados se ocorresse os seguintes fatores: a) flexão dos joelhos durante o voo; b) tronco e/ou cabeça inclinados à frente; c) aterrissagem com a planta do pé ao invés de tocar primeiro a ponta dos pés; d) movimento dos braços; e e) se não aconteceu à flexão até $90^{\circ}$.

\section{Variáveis do estudo}

As variáveis discutidas no presente estudo foram provenientes do percentual do uso do CAE, obtidas por meio de saltos verticais propostos por Bosco (8). Os valores do SJ e CMJ para a altura, potência de membros inferiores e atividade eletromiográfica foram, para os dois grupos do presente estudo (Handebol e Musculação), inseridas na seguinte equação: $\mathrm{CAE}=\{(\mathrm{CMJ}-\mathrm{SJ}): \mathrm{SJ} * 100\}$. Onde, CAE é o percentual de utilização do Ciclo Alongamento-Encurtamento, SJ é o salto Squat Jump e CMJ é o salto com o uso do contra movimento.

Com relação à atividade eletromiográfica foi mensurado o pico do percentual da CVIM na fase de impulsão de cada um dos saltos e esse valor foi inserido na equação do CAE citada acima. Desta forma, foi possível identificar o percentual de economia de energia neuromuscular realizado em função da utilização do CAE para cada um dos grupos (musculação e handebol) e cada músculo distinto (vasto lateral, bíceps femoral, reto femoral e gastrocnêmio medial). Para altura, potência e potência normalizada pela massa corporal foram inseridas os valores obtidos em cada tipo de salto.

\section{Análise estatística}

Os dados foram submetidos à estatística descritiva. Foi verificada a normalidade na distribuição dos dados por meio do teste de Shapiro-Wilk, a homogeneidade por meio do teste de Levene.

Para as comparações na caracterização dos grupos e nas variáveis analisadas, entre os grupos distintos, foi utilizado o teste $t$ de Student para amostras independentes. Nas comparações dentro do mesmo grupo foi utilizado o teste $t$ de Student pareado. O nível de significância para todos os testes foi de $5 \%$.

\section{Resultados}

Os resultados da caracterização dos indivíduos estão apresentados na Tabela 1, onde é possível observar que os grupos possuem características similares entre si.

$\mathrm{Na}$ Tabela 2 apresenta a estatística descritiva da atividade eletromiográfica dos músculos durante a realização dos saltos $\mathrm{SJ}$ e CMJ para os dois grupos ativos (handebol e musculação), além da probabilidade de significância (p-valor).

A Tabela 3 demonstra as médias e os desvios padrões da altura saltada e a potência dos saltos verticais, SJ e CMJ, para os grupos de handebol e musculação, além da probabilidade de significância (p-valor).

Tabela 1 - Caracterização da amostra segundo grupos: Grupo Handebol (GH) e Grupo Musculação (GM)

\begin{tabular}{|c|c|c|c|c|c|}
\hline & \multicolumn{2}{|c|}{ GH $(n=16)$} & \multicolumn{2}{|c|}{ GM $(n=12)$} & \multirow{2}{*}{$P$} \\
\hline & Méd & DP & Méd & DP & \\
\hline $\begin{array}{l}\text { Idade } \\
\text { (anos) }\end{array}$ & 21,13 & 3,59 & 19,50 & 3,34 & 0,234 \\
\hline $\begin{array}{l}\text { Estatura } \\
\text { (m) }\end{array}$ & 1,64 & 0,09 & 1,62 & 0,06 & 0,605 \\
\hline $\begin{array}{l}\text { Massa } \\
(\mathrm{kg})\end{array}$ & 63,98 & 8,46 & 61,16 & 9,77 & 0,421 \\
\hline $\begin{array}{l}\text { IMC } \\
\left(\mathrm{kg} / \mathrm{m}^{2}\right)\end{array}$ & 23,82 & 2,18 & 23,20 & 3,39 & 0,563 \\
\hline TP (anos) & 9,25 & 3,28 & 1,85 & 1,47 & $<0,001$ \\
\hline $\begin{array}{l}\text { TST } \\
\text { (horas) }\end{array}$ & 3,33 & 1,23 & 5,29 & 1,81 & $0,003 *$ \\
\hline
\end{tabular}


Tabela 2: Comparação da atividade eletromiográfica durante a realização do Squat Jump e do Counter Moviment Jump (CMJ) entre os grupos de handebol (GH) e de musculação (GM)

\begin{tabular}{|c|c|c|c|c|c|c|}
\hline & & \multicolumn{2}{|c|}{ GH $(n=16)$} & \multicolumn{2}{|c|}{ GM $(n=12)$} & \multirow[b]{2}{*}{$P$} \\
\hline & & $\begin{array}{c}\text { Média } \pm \text { Desvio } \\
\text { padrão }\end{array}$ & $\begin{array}{c}\text { \% de } \\
\text { utilização do } \\
\text { CAE }\end{array}$ & $\begin{array}{c}\text { Média } \pm \\
\text { Desvio padrão }\end{array}$ & $\begin{array}{c}\text { \% de } \\
\text { utilização do } \\
\text { CAE }\end{array}$ & \\
\hline \multirow[t]{2}{*}{$\begin{array}{l}\text { Vasto lateral } \\
\text { (\% da CVIM) }\end{array}$} & Squat & $122,20 \pm 118,86$ & $\begin{array}{c}-2,63 \\
\pm\end{array}$ & $117,40 \pm 133,09$ & $\begin{array}{c}13,32 \\
\pm\end{array}$ & \multirow{2}{*}{$\mathbf{0 , 0 1 5}$} \\
\hline & CMJ & $118,86 \pm 43,71$ & 15,96 & $133,09 \pm 60,71$ & 16,27 & \\
\hline \multirow{2}{*}{$\begin{array}{l}\text { Reto femoral } \\
\text { (\% da CVIM) }\end{array}$} & Squat & $107,61 \pm 38,52$ & $\begin{array}{c}-1,15 \\
\pm\end{array}$ & $106,72 \pm 34,15$ & $\begin{array}{c}1,75 \\
\pm\end{array}$ & \multirow{2}{*}{0,649} \\
\hline & CMJ & $104,95 \pm 37,18$ & 19,88 & $108,87 \pm 38,85$ & 10,35 & \\
\hline \multirow[t]{2}{*}{$\begin{array}{l}\text { Bíceps femoral } \\
\text { (\% da CVIM) }\end{array}$} & Squat & $115,63 \pm 138,83$ & $\begin{array}{l}-9,96 \\
\pm\end{array}$ & $71,27 \pm 56,23$ & $\begin{array}{l}9,10 \\
\pm\end{array}$ & \multirow[t]{2}{*}{0,112} \\
\hline & CMJ & $76,42 \pm 45,82$ & 35,37 & $73,71 \pm 48,26$ & 21,58 & \\
\hline \multirow{2}{*}{$\begin{array}{l}\text { Gastrocnêmio } \\
\text { medial } \\
\text { (\% da CVIM) }\end{array}$} & Squat & $121,61 \pm 41,09$ & 2,19 & $122,75 \pm 37,46$ & 4,43 & \multirow{2}{*}{0,622} \\
\hline & CMJ & $125,10 \pm 45,61$ & $\stackrel{ \pm}{ \pm} 13,11$ & $127,91 \pm 38,97$ & $\begin{array}{c} \pm \\
9,63\end{array}$ & \\
\hline
\end{tabular}

Squat: squat jump; CMJ: counter moviment jump; GH = Grupo Handebol; GM = Grupo Musculação; \% da CVIM: percentual da contração voluntária isométrica máxima; CAE: ciclo alongamento-encurtamento.

Tabela 3: Comparação do desempenho na altura alcançada na realização do Squat Jump e do Conter Moviment Jump para os grupos de handebol (GH) e de musculação (GM)

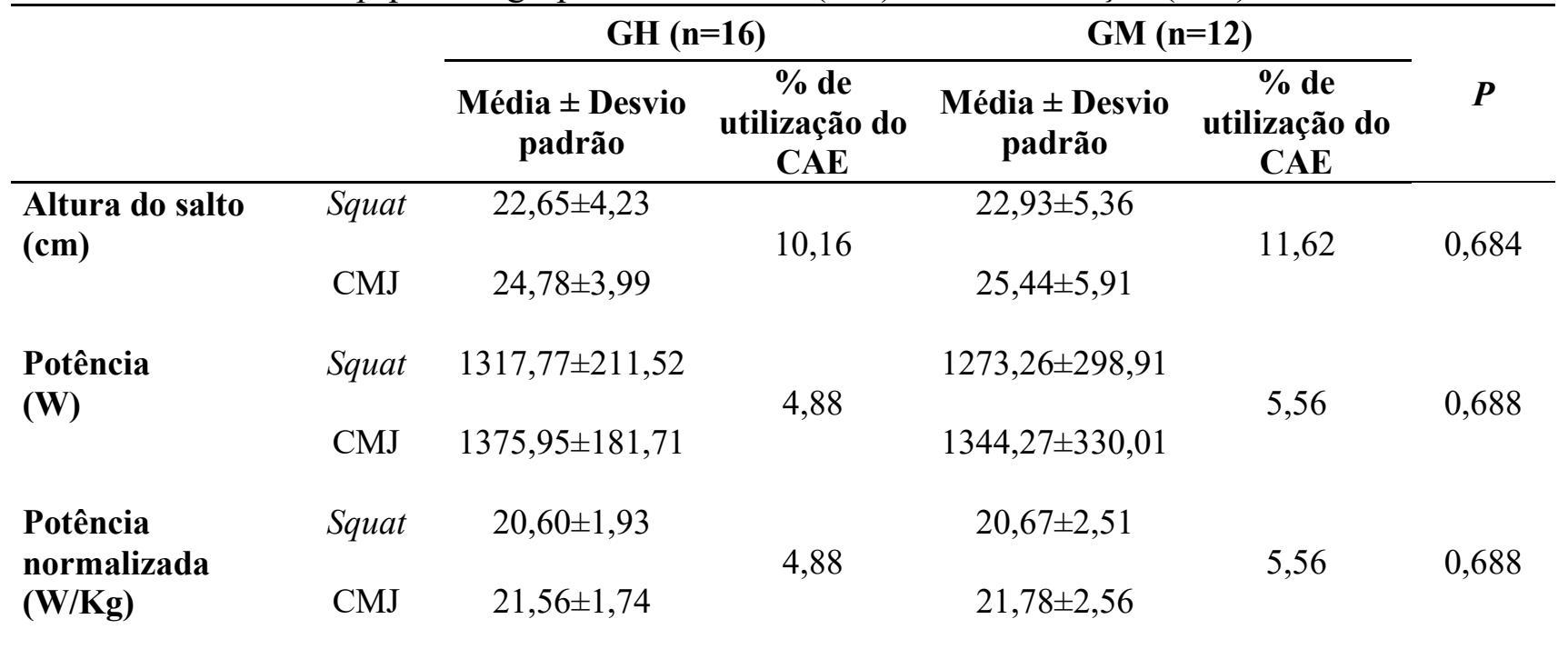

GH = Grupo Handebol; GM = Grupo Musculação; \% da CVIM: percentual da contração voluntária isométrica máxima; CAE: ciclo alongamento-encurtamento.

\section{Discussão}

$\mathrm{Na}$ maioria dos resultados do presente estudo é possível verificar a ausência de diferenças estatisticamente significativas entre os grupos GH e GM. Na maioria dos resultados do presente estudo é possível verificar a ausência de diferenças estatisticamente significativas entre os grupos GH e GM. Na Tabela 2, na qual estão apresentados os valores do sinal eletromiográfico e o percentual de ativação muscular envolvidos na relação entre $\mathrm{SJ}$ e CMJ, assim, percebe-se que apenas o músculo vasto lateral apresentou diferenças 
estatisticamente significativas entre os grupos do estudo.

Esses dados possibilitam o entendimento de uma maior economia eletromiográfica do músculo vasto lateral para atletas de handebol, pelo maior uso do ciclo Alongamento-Encurtamento (CAE) comparado com as praticantes de musculação. Enquanto as atletas de handebol diminuíram $2,63 \%$ de sua ativação para os saltos, as praticantes de musculação ampliaram 13,32\% a mesma variável para esse músculo.

Pode-se dizer então que essa diferença mostrada na estatística ocorreu possivelmente pelo fato de que as atletas de handebol, já têm em seu repertório motor um vasto conhecimento sobre movimentações e saltos, devido a grande utilização em jogo, e provavelmente sabem aproveitar a força elástica para ter maior economia de ativação, na realização dos saltos verticais. Esses achados são apoiados pelo princípio da especificidade, no qual Peñas e Graña (11) afirmam que, devido à duração da partida de handebol alcançar os 60 minutos e por realizarem diversos saltos, trotes, sprints e arremessos durante a mesma, caracterizando diferentes tipos de manifestações de resistência, fundamentando-se na capacidade de suportar um trabalho constante durante esse tempo, dá-se a necessidade de se trabalhar a potência aeróbica (11). Já a zona de treinamento de força caracteriza-se pela utilização de cargas elevadas, que podem alcançar de 80 a $100 \%$ de uma repetição máxima, implicando em menor número de repetições, com menor velocidade de execução (12).

Mas, baseando-se no princípio da especificidade existe diferença na economia de movimento para o músculo vasto lateral em relação aos demais músculos analisados?

As possíveis respostas para esse questionamento podem estar relacionadas às características morfológicas, fisiológicas e biomecânicas de cada um desses músculos em virtude de tempos de práticas e horas semanais de atividades distintas (Tabela 1) (13).

No caso do músculo reto femoral, ele apresenta maior proporção de fibras do tipo II que o vasto lateral, é composto por fibras fusiformes, tem menor área de secção transversal fisiológica e é biarticular, tendo função de distribuir o torque nas duas articulações e controlar a direção do movimento, apresentando uma estratégia de controle neural diferenciada dos músculos monoarticulares $(14,15)$. Já o músculo bíceps femoral, flexor do joelho, atua excentricamente na impulsão de saltos. $\mathrm{O}$ músculo gastrocnêmio medial apresenta um importante papel na produção de contração concêntrica na fase de saída do solo no salto vertical, onde ele realiza plantiflexão, gerando força para o voo e, assim obter um melhor desempenho (16).

Um dos fatores que devem ser levados em consideração para que os saltos sejam realizados com maior eficiência é a potência, onde a força relacionada com a velocidade do movimento pode gerar uma maior potência, em situações que o gesto motor precisa ser mais rápido para que a medida se dê pela força versus a velocidade que muitos esportes como o handebol, por exemplo, necessitam de um maior índice de potência na execução dos gestos motores da modalidade, o que a torna uma importante forma de manifestação de força (17).

As estruturas mais flexíveis deveriam ter maior complacência e, dessa forma, poderiam armazenar mais energia potencial elástica para ser utilizada como energia cinética no salto, uma musculatura que apresente um bom índice de alongamento (extensibilidade) tem potencializado o seu transporte de fluidos e sua capacidade mecânica de realizar contrações, permitindo um aproveitamento mais econômico da energia elástica (18).

Além disso, a arquitetura muscular é influenciada pela prática (13-21). Dessa forma, o treinamento de handebol se dá em várias angulações das articulações envolvidas nos saltos e nas situações de jogo, já o treinamento de musculação se dá em apenas um ângulo em suas movimentações de treino. Essa plasticidade neuromuscular é influenciada pelas características do treino. Com isso, a curva força versus o comprimento muscular deve ser diferente entre os grupos. O que significa que os testes avaliados neste estudo, aproximam-se das características do $\mathrm{GH}$ e, portanto, mais 
condizente com o pico de força na curva citada acima.

Já nas praticantes de musculação não se teve economia de movimento como as de handebol, isso pode vir do encurtamento muscular, que frequentemente ocorre, através do treinamento de força, o aumento de força com um proporcional aumento do tônus muscular, podendo vir a prejudicar a flexibilidade, gerado pelo excesso de hipertrofia onde são aumentadas as superfícies de contato entre os músculos e a pele, que pode limitar a flexibilidade em algum momento e conduzir a diminuição de seus níveis (22). Também pode ser considerado que as praticantes de musculação, não possuem em seu repertório motor a consciência corpórea adequada para a realização dos saltos e, por realizarem trabalho de força localizada, não tenham economia de movimento para obterem melhores números na realização dos saltos verticais, analisados no presente estudo.

\section{Conclusão}

É possível concluir, com bases nos achados do presente estudo, que as atletas de handebol sabem aproveitar a força elástica para terem maior economia de ativação em saltos verticais, por ser trabalhadas em seu treinamento diferentes angulações das articulações, o que mostrou que as praticantes de musculação não são econômicas possivelmente por causa de seu treinamento isolado.

São sugeridos novos estudos com a utilização da metodologia abordada no presente estudo, para que se possam ampliar os conhecimentos acerca dessa temática.

\section{Agradecimentos}

Os autores agradecem a Federação Gaúcha de Handebol e ao NEPE-FACOS.

\section{Declaração de conflito de interesses}

Não, nenhum conflito de interesses no presente estudo.

\section{Referências}

1. Vargas RP, Santi H, Duarte M, Cunha JR. Características antropométricas, fisiológicas e qualidades físicas básicas de atletas de handebol feminino. RBPFEX. 5(28): 352-362, 2010.

2. Fritzen AR, Castro I, Vignochi N, Navarro F. Treinamento intermitente e as características morfológicas, metabólicas e fisiológicas no handebol. Rev Bras de Prescrição e Fisiologia do Exercício. 4(23):449-456, 2010.

3. Trocóli TO, Furtado C. Fortalecimento muscular em hemiparéticos crônicos e sua influência no desempenho funcional. Res. Neurocienc. 17(4):336-41. 2009.

4. Coelho DB, Coelho LGM, Braga ML, Paolucci A, Cabido CMT, Junior JBF, Mendes TT, Prado LS, Garcia ES. Correlação entre o desempenho de jogadores de futebol no teste de sprint de $30 \mathrm{~m}$ e no teste de salto vertical. Motriz. 17(1):63-70, 2011.

5. Lazaridis SN, Bassa EI, Patikas D, Hatzikotoulas K, Lazaridis FK, Kotzamanidis CM. Biomechanical comparison in different jumping tasks between untrained boys and men. Pediatric Exercise Science. 25(1): 101-13, 2013.

6. De Luca CJ, Adam A, Wotiz R, Gilmore LD, Nawab SH. Decomposition of surface EMG signals. Journal of Neurophysiology Published. 96(3):1646-57, 2006.

7. Hermes HJ, Freriksa B, Disselhorst-Klugb C, Rau G. Development of recommendations for SEMG sensors and sensor placement procedures. J Electromyogr Kinesiol. Journal of Electromyography and Kinesiology. 10:361 - 374, 2000.

8. Corrêa F, Corrêa JCF, Martinelli JL, Oliveira AR, Oliveira CS. Reprodutibilidade da eletromiografia na fadiga muscular durante contração isométrica do músculo quadríceps femoral. Fisioter. Pesqui. 13(2): 46-52, 2006.

9. Cardoso J, et. al. Prado AI, Iriya HK, Santos ABAN, Pereira HM. Atividade eletromiográfica dos músculos do joelho em indivíduos com reconstrução do ligamento cruzado anterior sob diferentes 
estímulos sensório-motores: relato de casos. Fisioter. Pesqui.15(1):78-85, 2008.

10. Bosco C, Luhtanen P, Komi PV. A simple method for measurement of mechanical power in jumping. Eur J Appl Physiol Occup Physiol. 50: 273-82, 1983.

11. Penãs, CL, Granã PL. El entrenamiento de la velocidad en el balonmano. Revista Andebol, 28, 2000.

12. Kraemer WJ, Ratamess NA. Fundamentals of resistance training: progression and exercise prescription. Medicine and Science in Sports and Exercise, Madison.36(4):674-88, 2004.

13. Baroni BM, Franke RA., Rodrigues R, Geremia JM, Schimidt HL, Carpes FP, Vaz MA. Are the responses to resistance training different between the preferred and non-preferred limbs?. J Strength Cond Res. 30(3):733-738, 2016.

14. Pereira RH, Lima WP. Influência do treinamento de força na economia de corrida em corredores de endurance. Rev Bras de Prescrição e Fisiologia do Exercício. 4(20): 116-135, 2010.

15. Okano AH, Moraes AC, Bankoff ADP, Cyrino ES. Respostas eletromiográficas dos músculos vasto lateral, vasto medial e reto femoral durante esforço intermitente anaeróbio em ciclistas. Motriz. 11(1): 1124, 2005.

16. Fraga CHW, Bianco R, Gonçalves $\mathrm{M}$. Comparação do sinal EMG e das características da passada em diferentes protocolos de corrida incremental. Rev. bras. Educ. Fís. Esporte. 26(4):599-610, 2012.

17. Carvalho J, Borges GA. Exercícios de alongamento e suas implicações no treinamento de força. Caderno de $\mathrm{Ed}$. Fisica e Esporte. 3(2):67-78, 2001.

18. Rodrigues CEC, et al. Musculação: teoria e prática. 1985.

19. Baroni BM, Rodrigues R, Franke RA, Geremia JM, Rassier DE, Vaz MA. Time Course of Neuromuscular Adaptations to Knee Extensor Eccentric Training. Int J Sports Med, 34(10):904-11, 2013.
20. Jakobsen MD, Sundstrup E, Randers MB, Kjær M, Andersen LL, Krustrup P, Aagaard P. The effect of strength training, recreational soccer and running exercise on stretch-shortening cycle muscle performance during countermovement jumping. Hum mov sci. 31(4):970-86, 2012.

21. Herzog W. The biomechanics of muscle contraction: optimizing sport performance. Sport ortho trauma. 25(4): 286-293, 2009.

22. Padulo J, Tiloca A, Powell D, Granatelli G, Bianco A, Paoli A. EMG amplitude of the biceps femoris during jumping compared to landing movements. Springerplus. 2(520), 2013. 